\title{
Cerebrovascular disease in a newly diagnosed diabetic adolescent: case report
}

\begin{abstract}
Paediatric stroke is a rare occurrence with an incidence ranging from 1.3-13/100,000 of children. Little is known about the association of diabetic ketoacidosis and stroke. Also, the frequency of stroke in diabetic ketoacidosis is not defined as there are no reported cases. We report a case of a right-handed 14 year-old male adolescent referred to Ekiti State University Teaching Hospital (EKSUTH) from a peripheral hospital where he was managed for typhoid sepsis, however at presentation he had clinical and laboratory features of diabetic ketoacidosis. Diabetic ketoacidosis was managed with insulin infusion and recommended intravenous fluids. When the patient became euglycaemic and regained consciousness, he was noticed to have developed features of left hemispheric cerebrovascular disease which was confirmed with cranial $\mathrm{CT}$ and patient was managed as such till discharge from the hospital.
\end{abstract}

Keywords: cerebrovascular disease, type 1 diabetes, diabetic ketoacidosis
Volume 5 Issue I - 2017

Oluwayemi Isaac Oludare, ${ }^{1,2}$ Oke Julius

Oluwasola, ${ }^{2}$ Taiwo Adekunle Fesojaye ${ }^{2}$

'Department of Paediatrics, Faculty of Clinical Sciences, College of Medicine, Ekiti State University, Nigeria

${ }^{2}$ Department of Paediatrics, Ekiti State University Teaching Hospital, Nigeria

Correspondence: : Oluwayemi Isaac Oludare, Department of Paediatrics, Faculty of Clinical Sciences, College of Medicine, Ekiti State University,Ado-Ekiti, Ekiti State, Nigeria,Tel +2348034052536, Email issac.oluwayemi@eksu.edu.ng

Received: May 14, 2017 | Published: July 12, 2017
Abbreviations: EKSUTH, ekiti state university teaching hospital; CVD, cerebrovascular disease; DKA, diabetic ketoacidosis; CT, computerized tomography; IVF, intravenous fluid; Na, sodium; $\mathrm{K}$, potassium; $\mathrm{Cl}$, chloride

\section{Introduction}

Type 1 diabetes mellitus account for $10 \%$ of all diabetes, affecting over 15million people in the world and accounts for most cases of diabetes in childhood. ${ }^{1}$ One of the numerous complications of Type 1 diabetes mellitus is ketoacidosis, which occurs in about 20 to $40 \%$ of cases of newly diagnosed or older patients who omit their drugs or who are having intercurrent infection. ${ }^{1}$ Management of ketoacidosis requires careful attention to fluid, electrolyte and insulin therapy. It is readily complicated by cerebral edema, hypoglycemia and stroke and pneumomediastinum amongst other complications. The frequency of cerebral edema in ketoacidosis ranges from 0.39 to $0.5 \%$ with variable mortality and morbidity. ${ }^{2,3}$ The early signs of cerebral edema are headache, deterioration in consciousness, confusion and unexplained changes in heart rate, respiratory rate and blood pressure. Other neurological complications of diabetic ketoacidosis include hemorrhage, thrombosis and infections. ${ }^{4}$

\section{Case report}

We report a case of a right-handed 14year-old male adolescent referred to us from a peripheral center, where he was managed for typhoid sepsis having presented with history of progressive generalized body weakness and weight lost.

At presentation, the boy was unconscious with a Glasgow Coma Score of $9 / 15$, had fast and deep breathing, and random blood glucose too high for recording on glucometer.

We managed him as a case of newly diagnosed diabetes with ketoacidosis. Hyperglycemia was corrected with soluble insulin infusion at $0.1 \mathrm{unit} / \mathrm{kg} / \mathrm{hr}$ and intravenous fluid (IVF) at $8 \%$ free water deficit, plus maintenance. The initial IVF was normal saline till blood glucose reduced to less than $13.8 \mathrm{mmol} / \mathrm{L}$; then, it was changed to $5 \%$ Dextrose saline and insulin infusion reduced to 0.05 unit/ $\mathrm{kg} / \mathrm{hr}$. When RBG was less than $8.3 \mathrm{mmol} / \mathrm{L}$, insulin infusion was reduced to $0.025 \mathrm{unit} / \mathrm{kg} / \mathrm{hr}$. Patient became euglycaemic and regained consciousness within 44 hours of insulin therapy.

Patient was however noticed to have become aphasic, when he regained consciousness, and could not move his right upper and lower limbs. We managed as case of left hemispheric ischaemic stroke, the cranial CT (Figure 1) confirmed the diagnosis. The results of other investigations done are as follow: Packed cell volume was $31 \%$, urine culture \& sensitivity yielded no growth, electrolyte, urea and creatinine at admission and (the day after): $\mathrm{Na} 157.2$ (146), Cl 107.6 (133.3), K 5.5 (4.77) and bicarbonate 17 (24), urea 22.6 (21.48), Creatinine 507.4 (160) and Malaria parasite (+++). In addition to controlling blood glucose with insulin, patient was also given IV Ceftriaxone for probable sepsis and IV Artesunate for malaria. Patient was co-managed by Paediatric endocrinologist, Paediatric neurologist and Physiotherapist, Nutritionist, and Paediatric nurses until patient was discharged home.

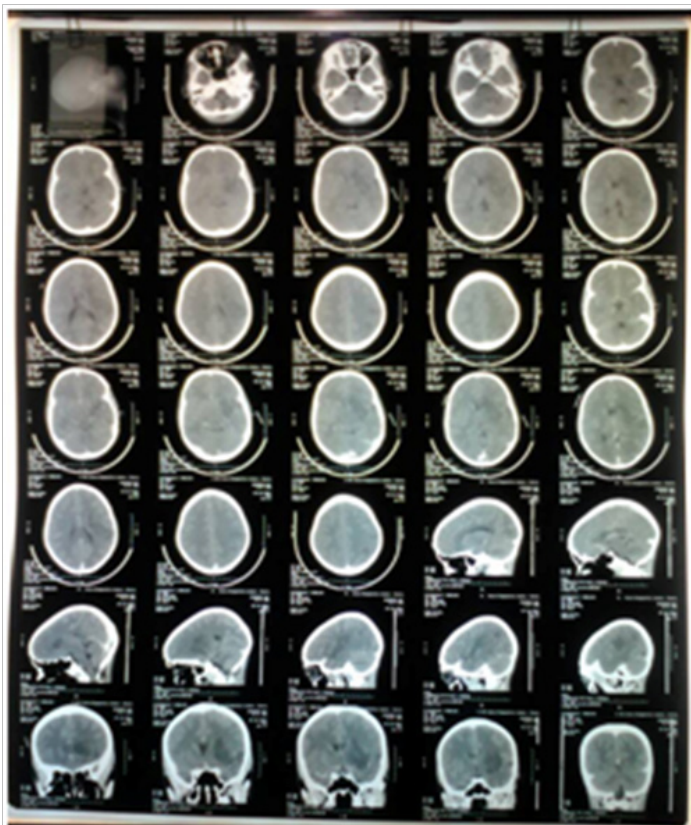

Figure I Cranial CT of index patient showing Left hemispheric ischaemic CVD. 


\section{Discussion}

Death due to thrombosis occurs in $80 \%$ of diabetic patients and $75 \%$ of these deaths is attributed to cardiovascular complications and the remaining $25 \%$ to cerebrovascular events and peripheral vascular complications. ${ }^{5}$ Pediatric stroke is a rare occurrence with incidence ranging from 1.3-13/100,000 of children. ${ }^{6}$ Little is known about the association of diabetic ketoacidosis and stroke. The frequency cerebrovascular disease in diabetic patients having DKA is not defined as there is no paper on such association however, there has being a few case reports.

Clinical features may not be specific; the commonest features in neonatal age group are seizure. ${ }^{8}$ Hemiparesis, dysphasia, visual disturbances are seen in older age group. The index patient was aphasic and had right hemiparesis when he regained consciousness.

The mechanism of ischemic stroke in children is not fully understood, however DKA is associated with systemic inflammatory response characterized by endothelial injury and coagulopathy. Vascular endothelium, the primary defense against thrombosis is abnormal in diabetes. Coagulation activation markers are elevated in diabetes, conversely, the level of the anticoagulant protein $\mathrm{C}$ is decreased. ${ }^{5,9}$ The fibrinolytic system responsible for removing clots is relatively inhibited in diabetes due to abnormal clot structures that are more resistant to degradation. ${ }^{5}$ There are also complement activations and elevated levels of inflammatory markers, and cytokines. ${ }^{9}$ Besides metabolic abnormalities other risk factors for ischaemic stroke include Sickle cell anaemia, congenital heart disease, coagulopathies and infection. The haemoglobin genotype of our patient is AA and he did not have congenital heart disease.

Ischaemic stroke is treatable with anticoagulation, careful attention to diet, prevention of infection, physiotherapy, good control of blood glucose and temperature.

Though we are limited in terms of facilities and fund in the management of the index case, the result was worth the effort. Patient has since been discharged home for follow-up and physiotherapy on out-patient basis.

Clinicians must be on the look-out for cerebrovascular accident when managing diabetes ketoacidosis otherwise many will be missed or managed as cerebral edema.

Clinicians in developing countries should also cultivate the habit of routinely checking the blood glucose of every patient. This will reduce the rate of misdiagnosing diabetic ketoacidosis (as occurred in index case that was previously wrongly managed for typhoid sepsis) and thereby enhance early management and recovery.

\section{Acknowledgement}

We sincerely appreciate all the Paediatric medical and nursing team that participated in the management of this patient.

Dr Oluwayemi I.O: participated in the management of the patient, conception, preparation and writing up of the manuscript

Dr Oke J.O: participated in the management of the patient and conception of the manuscript

Dr Taiwo A.F: participated in the management of the patient and prepared the manuscript

\section{Conflicts of interest}

We declare there is no conflict of interest and no financial support or grant for this work.

\section{References}

1. Alemzadeh R, Ali O. Type 1 Diabetes Mellitus (Immune mediated). In: Kliegman et al. Editors. Nelson text book of Paediatrics. $19^{\text {th }}$ edn, Philadelphia, USA: Elsevier/Saunders; 2011. p. 1968.

2. White PC, Dickson BA. Low morbidity and mortality in children with diabetic ketoacidosis treated with isotonic fluids. $J$ Pediatr. 2013;163(3):761-766.

3. Patel A, Singh D, Bhatt P, et al. Incidence, trends, and outcomes of cerebral edema among children with diabetic ketoacidosis in the United States. Clin Pediatr (Phila). 2016;55(10):943-951.

4. Rosenbloom AL. Intracerebral crises during treatment of diabetic ketoacidosis. Diabetes Care. 1990;13(1):22-33.

5. Carr ME. Diabetes mellitus; a hypercoagulable state. J Diabetes. 2001;15(1):44-54.

6. Mallick AA, O'Callaghan FJ. The epidemiology of childhood stroke. Eur J Paediatr Neurol. 2010;14(3):197-205.

7. Lindsay MP, Kapral MK, Gladstone D, et al. The Canadian stroke quality of care study: establishing indicators for optimal acute stroke care. CMAJ. 2015;172(3):363-365.

8. Kirton A, deVeber G. Cerebral palsy secondary to perinatal stroke. Clin Perinatol. 2006;33(2):367-386.

9. Hoffman WH, Burek CL, Waller JL, et al. Cytokine response to diabetic ketoacidosis and its treatment. Clin immunol. 2003;108(3):75-81. 